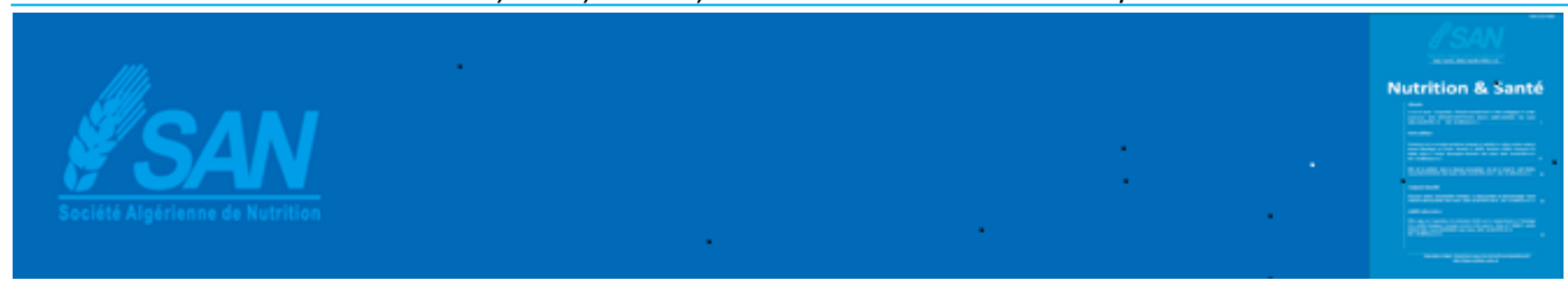

\title{
Composés bioactifs
}

\section{Structure unique, fonctionnalités multiples : La phycocyanine en biotechnologie}

One structure, multiple features: The phycocyanin in biotechnology

\section{Imene CHENTIR ${ }^{1,2}$, Moncef NASRI $I^{2}$}

${ }^{1}$ École Supérieure des Sciences de l'Aliment et des Industries Agroalimentaires (ESSAIA) Avenue Ahmed Hamidouche Route de Beaulieu, El Harrach 16200 - Alger. ${ }^{2}$ Laboratoire de Génie Enzymatique et de Microbiologie, Ecole Nationale d'Ingénieur de Sfax, Université de Sfax, B.P. 1173, Sfax 3038, Tunisie

Auteur correspondant : Chentir.imene@hotmail.com

Reçu le 20 avril 2020, Révisé le 06 juin 2020, Accepté le 12 juin 2020

Résumé La phycocyanine (PC) est une phycobiliprotéine, hydrosoluble non toxique, bioactive (anti-oxydantes, anti-inflammatoires, anti-tumorales, etc) isolée, principalement, à partir des cyanobactéries. En raison de ses propriétés, la PC est consi-dérée comme étant une biomolécule hautement exploitable en industrie et est devenue un axe de recherche crucial afin de promouvoir sa production et de maitriser ses applications biotechnologiques. Le présent article de synthèse a pour objectif de discuter les propriétés et applications basiques et récentes de la PC et de regrouper les données sur les différents aspects de la stabilisation et de la formulation de nano-particules de PC. Par ailleurs, une vue d'ensemble des principales caractéristiques struc-turales et des processus d'extraction, de purification de la PC sont également abordés. Les récents résultats de recherche scientifiques ont conclu que la PC est un additif, à la fois, fonctionnel et bioactif prometteur dans l'industrie, notamment, alimentaire, en tant que colorant, dans l'imagerie, en tant qu'agent fluorescent de marquage et dans le domaine pharmaceutique et nano-pharmaceutique, en tant que molécule et na-noparticules bioactives, particulièrement en oncologie, où elle a la capacité de bloquer la prolifération des cellules cancéreuses. La PC est, donc, une biomolécule prometteuse en pharmacologie et en médecine.

Mots clés: Phycocyanine, Cyanobactérie, Biotechnologie, Activité biologique, Effet antitumoral

Abstract Phycocyanine (PC) is a water-soluble, non-toxic and bioactive (antioxidant, antiinflammatory, antitumor, etc.) phycobiliprotein isolated, mainly, from cyanobacteria. Due to its several properties, PC is considered to be a rising biomolecule for Indus- 
trial exploitation, and has become an important research axis in order to promote its production, and optimize its biotechnological applications. The aim of this review article was to discuss the basic, and recent properties and applications of PC, and to bring together data on various aspects of PC stabilization, and PC nanopar-ticles formulation. In addition, an overview of the main structural characteristics and process-ses of PC extraction and purification were also discussed. The recent scientific research findings concluded that PC is a promising both functional, and bioactive additive in industry, especially, in food as a dye, in imaging as a fluorescent labeling agent, and in the pharmaceutical and nano-pharmaceutical field as a bioactive molecule and nanopar-ticles, particularly, due to it antitumor capacity. Phycocyanine is, thus, a promising bio-active molecules in pharmacological, and medical fields.

Keywords: Phycocyanin, Cyanobacteria, Biotechnology, Biological activity, Antitumoral effect

\section{Introduction}

La croissance rapide de la population humaine combinée aux perpétuels bouleversements économiques ainsi qu'au danger que peuvent avoir les produits chimiques sur la santé de l'Homme ont suscité l'intérêt de la communauté scientifique quant à l'exploration de nouvelles sources de molécules bioactives et thérapeutiques. La biomasse d'origine végétale, les plantes, notamment, a représenté depuis des siècles et, jusqu'à l'heure actuelle, le réservoir conventionnel de ces biomolécules. Ces dernières ont, largement, été utilisées en pharmacie, étant moins nocives que les différents principes actifs issus de l'industrie chimique $[1,2]$.

Au cours des années 1970, l'exploitation d'une nouvelle catégorie de biomasse émerge, celle des microalgues, incluant les cyanophycées ou microalgues bleues. Ces dernières présentent des avantages très intéressants, en comparaison avec les végétaux supérieurs. En effet, les microalgues requièrent peu d'eau, sont cultivables même sur les sols peu ou non fertiles et se caractérisent par une forte productivité et une bonne efficacité photosynthétique, offrant une récolte continue sur toute l'année. Ces avantages ont permis l'ouverture de nouveaux marchés propulsant, ainsi, les microalgues au rôle de candidates prometteuses pour la production de biomolécules d'intérêt, qui plus est, plus compétitives que celles extraites à partir de végétaux supérieurs $[3,4]$.

Similairement aux plantes, les microalgues synthétisent une large gamme de molécules bioactives, tels que les caroténoïdes, les chlorophylles, les acides gras, etc. Cependant, les microalgues bleues (cyanophycées), aux côtés des glaucophycées et des rhodophycées, sont les seules à produire des métabolites, uniques en leur genre, appelés phycobiliprotéines $[5,6]$.

D'un point de vue structural, les phycobiliprotéines sont des pigments accessoires à la photosynthèse, hydrophiles et non toxiques. Elles sont constituées d'un complexe de chaînes polypeptidiques liées de manière covalente à un phycobiline (pyrrole), jouant le rôle de chromophore hautement fluorescent. Dépendamment de la couleur de la fraction de phycobiline, les phycobiliprotéines se subdivisent en trois classes: la phycocyanine (chromophore bleu), la phycoérythrine (chromophore rouge) et l'allophycocyanine (chromophore jaunâtre) [7]. La phycocyanine est, actuellement, la phycobiliprotéine la plus produite à l'échelle industrielle, à partir de la spiruline [8] mais, aussi, la plus appliquée dans le domaine pharmaceutique, nutraceutique et biomédical, en tant qu'extrait issu des microalgues, derrière les caroténoïdes $[9,6]$. Toutefois, son exploitation demeure timide et émergente, comparativement aux extraits végétaux conventionnels. Par ailleurs, de par sa structure unique et son degré de pureté variable, la PC est douée d'une large gamme d'activités biologiques. De nombreuses études ont mis en évidence différentes bioactivités de la PC dont l'activité anti-oxydante, anti-inflammatoire, anti-tumorale, anti-microbienne, neuroprotectrice, hépato-protectrice, etc... [10], faisant d'elle, une molécule thérapeutique très prometteuse en médecine et en pharmacie [11]. De plus, d'autres travaux ont indiqué, qu'en raison de la présence du chromophore, la PC peut être utilisée en tant que marqueur fluorescent dans le diagnostic médical ou le diagnostic thérapeutique servant, ainsi, de substitut à plusieurs agents artificiels toxiques $[9,12]$. Bien que les travaux de recherche dans le domaine de la phytothérapie aient, continuellement, fait appel à de nouvelles sources rentables et économiques de 
biomolécules, la phytothérapie moderne, elle, implique la mise en place de procédés d'extraction rentables, simples et conjugués à de procédés de stabilisation qui permettent d'augmenter la durée de vie de la biomolécule d'intérêt assurant, ainsi, toute l'efficacité de son activité [13]. Par conséquent, les procédés d'extraction, de purification et de stabilisation de la PC à partir des microalgues, en général, n'échappent pas à cette règle et constituent un point crucial au carrefour de ses différentes applications pharmacologiques [14-16].

Cet article de synthèse présente un aperçu cohérent et une vue d'ensemble condensée des connaissances récentes sur les propriétés biologiques et pharmacologiques de la $\mathrm{PC}$, mettant la lumière sur les avancées récentes concernant la nano-administration de la PC. Par ailleurs, cet article de synthèse discute les caractéristiques générales, les techniques de production, de purification et de stabilisation de la PC, qui conditionnent son application.

\section{Caractéristiques générales de la phycocyanine}

La PC est une chromoprotéine, appartenant à la famille des phycobiliprotéines, assemblées sous une structure macromoléculaire régulière, appelée «phycobilisome» se trouvant au niveau de la membrane extérieure du thylacoïde. Au niveau du phycobilisome, la PC se localise à sa périphérie sous forme de 6 tiges portées par le cœur central composé d'allophycocyanine, fonctionnant comme le pigment construisant un pont entre le phycobilisome et les lamelles photosynthétiques du thylacoïde [17] (Fig. 1A).
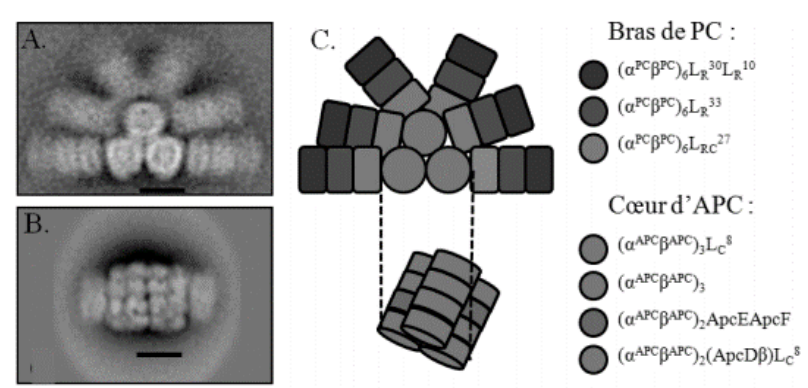

Fig. 1. Phycobilisome de l'espèce synochocystis PCC 6833 Image en microscopie électronique indiquant une vue de face $A$ et $d^{\prime}$ en haut $B$ du phycobilisome de synochocystis PCC 6833 à une échelle de $10 \mathrm{~nm}$ [17]. C Schéma représentatif du même phycobilisome avec projection orthogonale du cœur d'allophycocyanine. $P C$ : Phycocyanine. APC : Allophycocyanine.

D'un point de vue structural, la PC est composée d'une protéine (une chaine polypeptidique), liée à un chromophore, appelé phycocyanobiline, de couleur bleu intense, par des résidus cystéine à travers une liaison thioether (Fig. 2) [18]. La composante apopro- téique est constituée de deux sous-unités homologues $\alpha$ et $\beta$, de type globuline, dont la masse moléculaire est comprise entre 12 et $20 \mathrm{kDa}$ et entre 15 et $22 \mathrm{kDa}$, respectivement [7]. Ces deux sous-unités forment le monomère de la PC. En revanche, la composante phycocyanobiline correspond à des groupements prosthétiques linéaires isomériques de type térapyrrole [19].

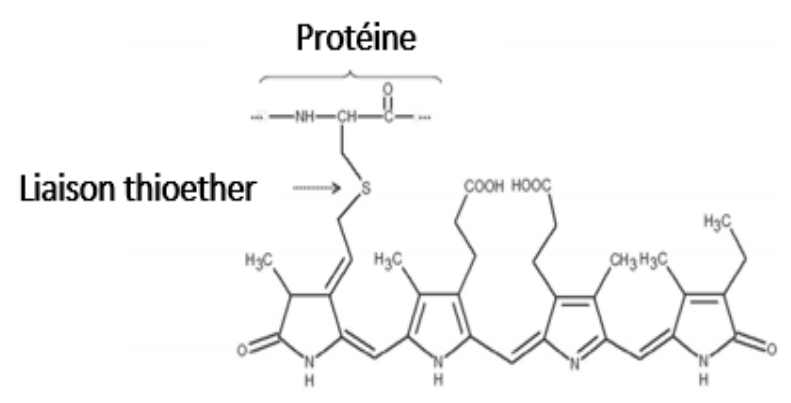

Fig. 2. Structure chimique du chromophore phycocyanobiline chez la phycocyanine d'Arthrospiraplatensis.

D'un point de vue optique, la PC affiche une bande d'absorption maximale entre 615 et $620 \mathrm{~nm}$ [18], avec un coefficient d'extinction molaire de 770000 $\mathrm{M}^{-1} \mathrm{~cm}^{-1}$, alors que son pic maximal de fluorescence se situe à $647 \mathrm{~nm}[19,20]$. Le spectre infrarouge (IR) de la $P C$ native (pureté égale à 1,24 ) présente une bande entre $3600 \mathrm{~cm}^{-1}$ et $3000 \mathrm{~cm}^{-1}$, attribuée aux élongations $\mathrm{O}-\mathrm{H}$ chevauchant les vibrations $\mathrm{N}-\mathrm{H}$ relié à l'amide $A$ à $3300 \mathrm{~cm}^{-1}$. Les bandes principales dans le profil IR de la PC sont observées à $1652 \mathrm{~cm}^{-1}$ et à 1541 $\mathrm{cm}^{-1}$ et sont associées à l'amide I et l'amide II, respectivement, de la composante protéique. De plus, deux autres bandes caractéristiques du chromophore de la PC peuvent être observées aux alentours de2914 $\mathrm{cm}^{-1}$ et $2840 \mathrm{~cm}^{-1}$, associées aux vibrations C$\mathrm{O}-\mathrm{H}$ et aux élongations symétriques et asymétriques $\mathrm{C}-\mathrm{H}$, respectivement [14]. Le $\mathrm{pH}$ isoélectrique de la $\mathrm{PC}$, qui dépend, principalement, de sa pureté, varie de 3,0 à $6,4[21,14]$.

\section{Production et productivité de la phycocyanine}

Le marché mondial connait, actuellement, une demande croissante et continue en PC. En effet, l'estimation de demande de la PC passera de 122,3 millions de dollars en 2018 à 233 millions de dollars en 2028 [22] justifiant, ainsi, le nombre important de travaux scientifiques, au cours de ces dernières années sur la culture des microalgues bleues, en vue de maximiser la production de la PC, à grande échelle [23]. A travers ces études, deux stratégies de culture de microalgues blues en ressortent, dont le but est de 
maximiser les rendements en PC dans la biomasse cultivée. La première stratégie concerne l'accumulation de la PC à travers l'augmentation de la densité de la biomasse produite, en manipulant son mode trophique. Trois modes sont, alors, adoptés: (i) le mode photoautotrophique, utilisant le soleil comme source d'énergie, (ii) le mode hétérotrophique, utilisant du carbone organique comme source d'énergie et (iii) le mode mixotrophique qui est la combinaison des modes photoauto- et hétérotrophique [10]. La seconde stratégie implique une maximisation ciblée du rendement de la PC, en exposant ses cultures aux conditions de stress biotiques et/ou abiotiques. Différentes études ont été entreprises afin d'évaluer l'effet de certaines conditions de stress sur le taux de PC produite. Ainsi, plusieurs tendances de maximation de la PC ont été décrites dans la littérature. Le Tableau I regroupe les conditions biotiques et abiotiques, visant à optimiser la production de la PC. II ressort de l'analyse de ce tableau que, vraisemblablement, il n'existe pas de conditions standards à la production de la PC. La variation de l'intensité de la lumière, la concentration des sels de culture, tels que le $\mathrm{NaCl}$ ou le $\mathrm{NaNO}_{3}, \mathrm{CaCl}_{2}, \mathrm{Na}_{2} \mathrm{CO}_{3}, \mathrm{MgSO}_{4}$ ou l'ajout $\mathrm{d}^{\prime}$ une source de glucose peut orienter le métabolisme vers la production induite de la PC.

Quelques études ont été entreprises afin d'évaluer l'effet de certaines conditions de stress sur le taux de PC produite. Chez la souche Arthrospirasp., I'utilisation de concentrations élevées en $\mathrm{NaCl}$ (jusqu'à 0,6 M) stimule, également, la production de la PC, atteignant 17,50\% [24]. Chez la souche saharienne Arthro- spira sp. cultivée suivant le processus de culture en deux phases, la teneur en PC a atteint les $16,09 \%$ du poids sec, au bout de 3 jours de culture, sous une lumière faible $\left(10 \mu \mathrm{mol}\right.$ de photons $\left.\mathrm{m}^{2} / \mathrm{s}\right)$ combinée à $0,33 \mathrm{M} \mathrm{NaCl}, 0,5 \mathrm{~g} / \mathrm{L}$ de $\mathrm{NaNO}_{3}$ et $2,68 \mathrm{~g} / \mathrm{L}$ de $\mathrm{K}_{2} \mathrm{HPO}_{4}$, [3]. Chez la cyanobactérie Limnothrix sp. 37-2-1, I'utilisation d'une intensité de lumière égale à $25 \mu \mathrm{mol}$ de photons $\mathrm{m}^{2} / \mathrm{s}$ permet d'obtenir une production de PC de 16\% [27]. En revanche, chez Euthalothèce sp. l'utilisation de $0,1 \mathrm{~g} / \mathrm{L}$ de $\mathrm{MgSO}_{4}, 1,67 \mathrm{~g} / \mathrm{L}$ de $\mathrm{NaNO}_{3}$, et $10 \%$ d'acide acétique, de $\mathrm{CaCl}_{2}$ et $\mathrm{Na}_{2} \mathrm{CO}_{3}$ induit une production de $45 \%$ de PC [28]. Par ailleurs, la productivité journalière de PC chez $A$. platensis, cultivée à grande échelle en mixotrophie $(114,74$ $\mathrm{mg} / \mathrm{L}$ de mélasse) en présence de 0,192 $\mathrm{g} / \mathrm{L}$ d'urée, atteint les $21,25 \mathrm{mg} / \mathrm{L} / \mathrm{J}$ [25]. De même la culture d' $A$. platensis en mixotrophie, par ajout de $0,1 \%$ de glucose, a permis une production de $38 \%$ de $\mathrm{PC}$ après 10 jours de culture [26].

\section{Extraction et purification de la phycocyanine}

Les différentes applications de la PC sont, fortement, liées à sa pureté qui, par définition, est le ratio d'absorbance (A) A620/A280, où l'absorbance à $620 \mathrm{~nm}$ et à $280 \mathrm{~nm}$ correspond, respectivement, à l'absorbance maximale de la PC et à celle des protéines totales. Se référant aux données de la littérature, il a été établi qu'un ratio d'absorbance A620/A280 inférieur ou égal à 0,7 , la $P C$ est considérée comme étant de qualité alimentaire; de 0,7 à 3,9 , la PC est de qualité réactive ; alors qu'un ratio supérieur à 4,0

Tableau I. Conditions de production de la phycocyanine par les microalgues blues

\begin{tabular}{|c|c|c|c|}
\hline Souche & Mode de culture & Rendement/productivité & Références \\
\hline Arthrospira sp. & Photoautrophie ; milieu Zarrouk avec $0,6 \mathrm{M} \mathrm{NaCl}$ & $17,50 \%$ & [24] \\
\hline Arthrospira sp. & $\begin{array}{l}\text { Photoautotrophie; culture en deux phases de } 3 \text { jours; } \\
\text { Lumière : } 10 \mu \text { mol de photons } \mathrm{m}^{-2} \mathrm{~S}^{-1} \text {; Milieu Zarrouk avec } \\
0,5 \mathrm{~g} / \mathrm{L} \mathrm{NaNO} \mathrm{Na}_{3}+11,7 \mathrm{~g} / \mathrm{L} \mathrm{NaCl}+2,68 \mathrm{~g} / \mathrm{LK}_{2} \mathrm{HPO}_{4} \text {. }\end{array}$ & $16,09 \%$ & [3] \\
\hline A. platensis & $\begin{array}{l}\text { Mixotrophie ; Milieu Bengladech avec } 0,192 \mathrm{~g} / \mathrm{L} \text { urée }+ \\
114,74 \mathrm{mg} / \mathrm{L} \text { mélasse ; Lumière : } 45 \mu \mathrm{mol} \text { de photons } \mathrm{m}^{-2} \mathrm{~s}^{-1} \text {. }\end{array}$ & $21,25 \mathrm{mg} / \mathrm{L} / \mathrm{jour}$ & [25] \\
\hline A. platensis & $\begin{array}{l}\text { Photoautotrophie; milieu Zarrouk; Lumière : LED jaune de } \\
3000 \text { Lux. }\end{array}$ & $24,20 \%$ & [26] \\
\hline A. platensis & $\begin{array}{l}\text { Mixototrophie; milieu Zarrouk }+0,1 \% \text { glucose; Lumière } \\
\text { LED jaune de } 3000 \text { Lux. }\end{array}$ & $38 \%$ & [26] \\
\hline $\begin{array}{l}\text { Limnothrix sp. 37- } \\
\quad 2-1\end{array}$ & $\begin{array}{l}\text { Photoautotrophie; milieu BG-11; Lumière : } 25 \mu \mathrm{mol} \text { de } \\
\text { photons } \mathrm{m}^{-2} \mathrm{~s}^{-1} \text {. }\end{array}$ & $16 \%$ & [27] \\
\hline Euhalothèce sp. & $\begin{array}{l}\text { Photoautotrophie; milieu BG-11 avec } 0,1 \mathrm{~g} / \mathrm{L} \mathrm{MgSO} 4,1,67 \\
\text { g/L NaNO} \\
\text { photons } \mathrm{m}^{-2} \mathrm{~S}^{-1} \text {, }\end{array}$ & $45 \%$ & [28] \\
\hline Synochocystis sp. & $\begin{array}{l}\text { Photoautotrophie; milieu } \mathrm{BG}-11 \text { avec } 0,058 \mathrm{~g} / \mathrm{L} \mathrm{CaCl}_{2}, 0,11 \\
\mathrm{~g} / \mathrm{L} \mathrm{Na} \mathrm{CO}_{3} \text {; Lumière: } 75 \mu \mathrm{mol} \text { de photons } \mathrm{m}^{-2} \mathrm{~S}^{-1} \text {. }\end{array}$ & $12 \%$ & [29] \\
\hline
\end{tabular}


permet de classer la PC en qualité analytique ou phar -maceutique [30]. Ainsi, la purification de la PC est un point crucial reliant sa production et son application ; plus la pureté est grande plus son application touche des domaines pointus, tels que la pharmacologie ou le diagnostic médical $[6,9]$. Différentes méthodes physiques et chimiques optimisées, depuis la rupture des cellules à la purification de la PC, ont été développées et ces trois types de pureté de la PC peuvent être distingués.

Par ailleurs, bien que les méthodes utilisées diffèrent entre elles, ces dernières se basent sur le même principe, à savoir : i) la lyse cellulaire et récupération de l'extrait brut de PC, ii) purification partielle de la PC, et iii) purification poussée de la PC, moyennant les différentes variantes de la chromatographie.

La lyse cellulaire consiste à perturber l'intégrité physique des cellules afin de faciliter la libération de la PC contenue dans les thylacoïdes vers l'extérieur. Cette étape s'effectue, principalement, par l'alternance de cycles successifs de congélation-décongélation, combinés ou non à l'ultrasonication [31, 32]. L'application d'un choc osmotique via l'utilisation de tampons d'extraction, types phosphate, acétate, $\mathrm{NaCl}$ ou $\mathrm{CalC}_{2}$, avant l'étape de congélation-décongélation permet d'optimiser la cinétique de libération de la PC [18]. Par ailleurs, d'autres méthodes de lyse cellulaire, moins conventionnelles ont, également, été décrites dans la littérature. Ces dernières impliquent l'utilisation de l'acide hydrochloridrique [33] et d'enzymes, telles que les lysozymes [34], les cellulases, et les pectolyases [35].

En outre, l'utilisation d'un pulse électrique [36], des microondes [37] et des fluides supercritiques [38] a également été décrite pour la libération de la PC. La $\mathrm{PC}$ obtenue à cette étape est, en général, de grade alimentaire.

Le fractionnement et la purification: Afin de passer $d^{\prime}$ 'une $P C$, de grade alimentaire, à celle de grade réactif et analytique, nombreuses techniques ont été décrites dans la littérature. Le Tableau II résume les principales méthodes de purification de la PC.

La méthode la plus répandue, à grande échelle, consiste en différentes phases de fractionnement par précipitation, à l'aide du sulfate d'ammonium $\left(\left(\mathrm{NH}_{4}\right)_{2} \mathrm{SO}_{4}\right)$ allant d'une saturation de 20 à $65 \%$ $(\mathrm{m} / \mathrm{v})$,afin de précipiter la PC $[12,39]$. L'étape de fraction-nement peut être suivie d'une étape de dialyse à un seuil de coupure n'excédant pas les 14 $k D a$, servant à éliminer, uniquement, l'agent précipitant et les protéines de contamination améliorant, ainsi, la pureté de la PC [39]. Dans le but d'améliorer, davantage, le degré de pureté de la PC et atteindre des ratios supérieurs à 3 , différentes étapes de purification par chromatographie de perméation sur gel (Sephadex G-100) ou chromatographie échangeuse d'ions $[39,40]$ ont été adoptées. Le recours à différents adsorbants, tels que le charbon activé et le chitosane [30], l'hydroxyapatite [18] ou le PEG/ phosphate d'ammonium $[40,41]$ a, également, été décrit, servant d'étape de prétraitement de l'extrait de PC. Toutefois, la combinaison de la chromatographie échangeuse d'ions (DEAE-Sepharose) et la chromatographie à perméation sur gel améliore la pureté de la PC pouvant, alors, atteindre des indices de pureté égales à 6 .

En plus des méthodes chromatographiques, plusieurs travaux ont décrit la possibilité de purification de la PC, moyennant l'ultrafiltration à un seuil de coupure de $50 \mathrm{kDa}[41,12]$.

Les aspects de la stabilité et la stabilisation de la phycocyanine

A l'état natif, la PC présente une conformation trimérique ou hexamèrique après son extraction et se caractérise par une couleur bleue [43]. Néanmoins, la manipulation post-extraction de la PC peut provoquer la dénaturation complète ou partielle de sa conformation ce qui se traduit par son blanchiment. Selon la littérature, les facteurs, connus pour être les plus dénaturants, sont la température et la variation $\mathrm{du} \mathrm{pH}$. En effet, plusieurs travaux ont attesté que $50 \%$ de la $P C$ est dénaturée à des températures dépassant les $50^{\circ} \mathrm{C}$, pour une durée de traitement allant de 15 à $45 \mathrm{~min}[44-47,14]$. Cette dénaturation consiste, structuralement, en une altération de la structure secondaire de l'apoprotéine et l'altération de la liaison thioether entre l'apoprotéine et le chromophore, ce qui provoque le blanchiment de sa couleur bleue et la perte de la molécule $[48,14]$. Par ailleurs, le $\mathrm{pH}$ est un autre facteur qui affecte la stabilité de la PC. Dans une plage de pH allant de 5.0 à 7.0, la PC présente une stabilité maximale [46]. A des valeurs de $\mathrm{pH}$ inférieures à 5.0, la composante protéique est, partiellement, dépliée, ce qui se traduit par une faible solubilité, accompagnée par l'apparition d'un précipité, probablement, due au point isoélectrique, ainsi que par une faible absorbance à $620 \mathrm{~nm}$ [14]. En revanche, sous des conditions alcalines élevées, la solubilité de la PC est maximale, alors quel'absorbance à $620 \mathrm{~nm}$ est réduite; ceci pourrait être attribué à l'état électronique du chromophore et de la composante protéique, conduisant à leur dissociation, ce qui provoque le blanchiment de la PC [49]. 
Tableau II. Méthode de purification de la phycocyanine extraite à partir des microalgues blues

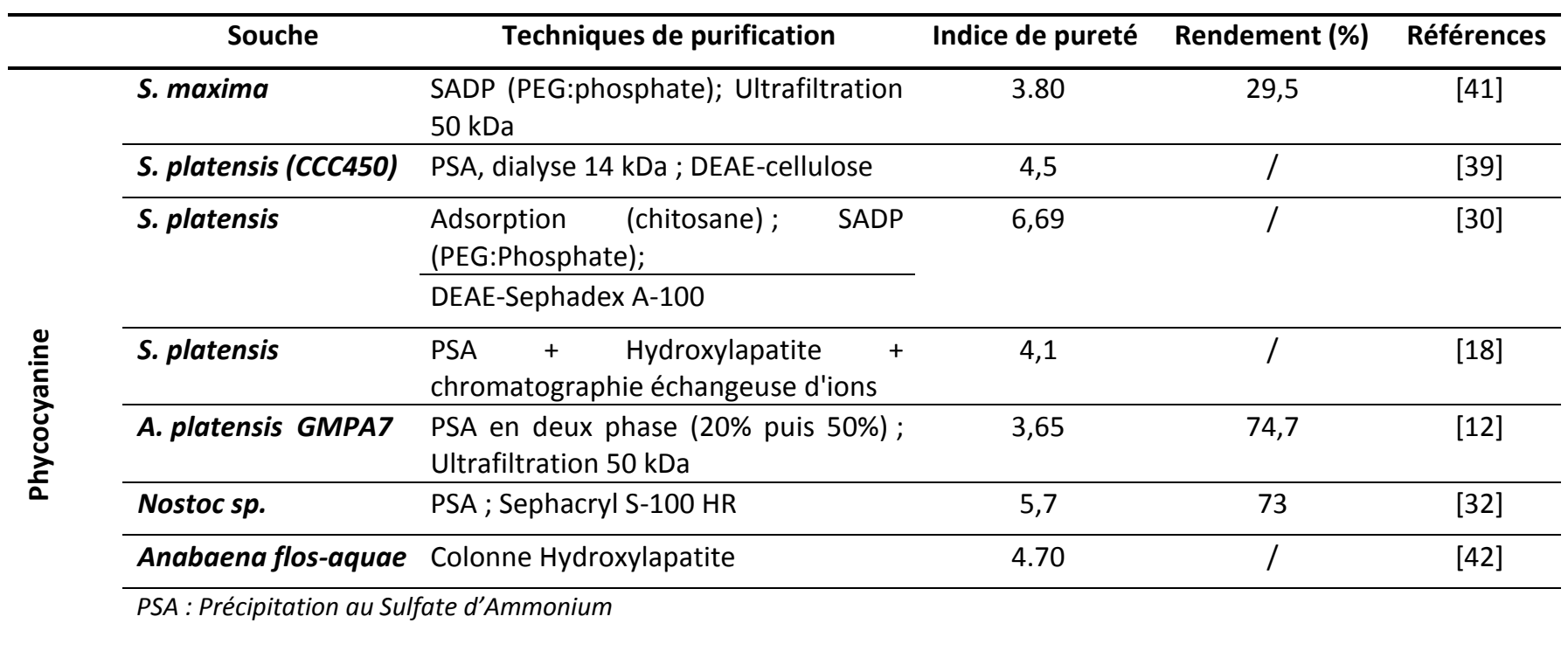

Plusieurs études ont montré qu'à une valeur de $\mathrm{pH}$ égale à 6.0, la PC est, entièrement, fonctionnelle, joignant une bonne solubilité et une haute absorbance à $620 \mathrm{~nm}[45,46,14]$. En raison de l'effet dénaturant que peut exercer l'environnement extérieur auquel la PC pourrait être exposée, lors de son traitement industriel, ainsi que les éventuelles réactions qui peuvent en découler, le besoin de développer un système de protection de la PC est devenu un axe de recherche très important, au cours de ses dernières années. En effet, la thermosensibilité de la PC limite son utilisation. Plusieurs travaux ont signalé que la thermostabilisation de la PC pourrait être renforcée par la modification de son environnement chimique via l'ajout de divers additifs, tels que les sucres (glucose, saccharose, fructose) $[45,50]$, les polyols (sorbitol, PEG-4000) [44,14], les sels ( $\mathrm{NaCl}$ [45]et les acides faibles (acides ascorbiques ou acide citrique) $[45,51]$.Ces derniers sont utilisés et admis en industries alimentaires et pharmaceutiques. Cependant, la stabilisation de la PC est, le plus souvent, abordée, de façon empirique, les mécanismes impliqués dans la stabilisation de la PC, moyennant les agents cités plus hauts n'étant pas encore bien élucidés. Chentir et al., [14] ont étudié ce mécanisme et ont conclu que cette thermostabilisation serait due à l'emplacement de l'agent stabilisant à la surface de la PC qui, suite à l'établissement de liaisons hydrogène, forme une couche protectrice responsable du ralentissement de l'interconversion et de la relaxation de l'apoprotéine de la PC préservant, ainsi, sa structure secondaire. Dans cette même optique, au cours de la dernière décennie, la stabilisation de la PC a été abordée sous un aspect, autre que chimique, incluant sa complexation et sa micro- et nano-encapsulation, sous forme de microcapsules, nanofibres ou liposomes, etc. [5255]. Divers polymères, tels que l'alginate de sodium, le chitosane, le maltodextrine, le carraghénane, la pectine, ont été décrits comme étant très efficients, quant à leurs capacités à encapsuler et à stabiliser la PC quand celle-ci est exposée à des variations de température et de $\mathrm{pH}$ mais, également, à l'action des enzymes protéolytiques [56-58].

\section{Applications biotechnologiques de la PC}

Les travaux de recherche portant sur les applications biotechnologiques de la PC n'ont, concrètement, été entamés, qu'au début des années 90, lorsque la purification et l'élucidation de sa structure ont été bien maitrisées. Cette présente section évoque les principales applications biotechnologiques de la PC (Fig. 3).

\section{Applications agroalimentaires de la PC}

En industrie agroalimentaire, la PC est utilisée, principalement, comme colorant naturel remplaçant les colorants artificiels toxiques pour la santé. En 2013, la PC est le premier colorant naturel à être autorisé par la Food and Drug Administration pour être utilisé par I'industrie alimentaire due à ses propriétés non cancérogènes [10]. Des études ont décrit les propriétés fonctionnelles de la PC : pouvoir moussant [14], pouvoir émulsifiant [59] et pouvoir améliorateur de viscosité des émulsions [60]. 
En outre, la PC a été incorporée dans différentes formulations alimentaires, incluant les boissons type énergisantes ou anti-oxydantes, les confiseries et les produits laitiers (glaces et desserts lactés) [61].

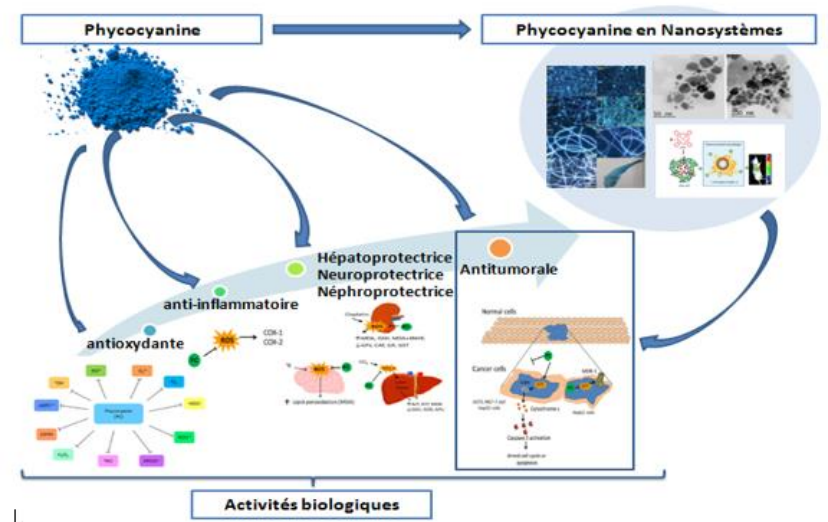

Fig. 3. Principaux effets biologiques de la PC et des nanoparticules de la PC [21]

Récemment, Chentir et al., [62] ont montré que la PC, obtenue à partir de la cyanobactérie Saharienne Arthrospira sp. pourrait être ajoutée, comme agent fonctionnel et bioactif, dans un bio-emballage alimentaire, à base de gélatine. L'ajout de la PC a permis l'obtention d'un emballage bioactif, à effet UVbarrière, antioxydant et antibactérien, permettant de mieux conserver les produits alimentaires.

\section{Applications de la phycocyanine en tant qu'a- gent fluorescent}

Une fois extraite, la PC se désintègre et perd ses accepteurs naturels d'énergie et, par conséquent, devient hautement fluorescente [30]. La fluorescence est proportionnelle à l'assemblage structural de la PC en solution. En effet, une solution, contenant des hexamères et des trimères de $P C$, est plus fluorescente qu'une solution contenant de la PC dissociée en monomère [30].Par ailleurs, la composition en acides aminés des chaînes polypeptidiques ainsi que la présence des groupements carboxyles permettent à la PC de se lier facilement à d'autres molécules [30]. Ainsi, des sondes fluorescentes, à base de PC, conjuguée à d'autre protéines, telles que les immunoglobines, la protéine $\mathrm{A}$ et l'avidine ont été développées et, largement, utilisées en histochimie, en microscopie à fluorescence, en cytométrie de flux, le tri cellulaire à fluorescence, les dosages immunologiques par fluorescence et le marquage des protéines et des anticorps [9]. Simis et al., [63] ont signalé que la PC, partiellement purifiée, pourrait être utilisée comme substitut au bromure d'éthidium, habituellement utilisé, pour des analyses immunologiques et coloration de I'ADN. Badakere et al., [64] ont indiqué la possibilité de coloration et de marquage de globules rouges, de globules blancs, de plaquettes, des lymphocytes, de cellules nucléées et de l'ADN génomique par la PC. De plus, la fluorescence de la PC a été exploitée in vivo, par mesure directe de fluorescence, pour la détection de cyanobactéries toxiques dans les eaux potables et la télédétection des cyanobactéries dans des milieux naturels [65].

\section{Applications biologiques et pharmaceutiques de la PC}

Le potentiel biologique et pharmaceutique de la PC a été rapporté par plusieurs études. Les Tableaux III et IV présentent un récapitulatif de ses différents effets biologiques et pharmaceutiques.

\section{Effet antioxydant de la PC}

Le déséquilibre entre les systèmes antioxydants et la production de dérivés réactifs à l'oxygène est appelé " stress oxydatif ». Ce dernier peut être à l'origine de diverses maladies, notamment, l'athérosclérose, les maladies neuro-dégénératives, le cancer, le diabète, les maladies inflammatoires, ainsi que le processus de vieillissement [66]. En 1998, l'activité antioxydante de la PC a été décrite, pour la première fois, par Romay et al., [67]. La PC serait capable de piéger plusieurs radicaux libres, incluant l'hydroxyle $(\bullet \mathrm{OH})$, le radical alcoxyle $(\mathrm{RO} \bullet)$ et l'anion superoxyde $\left(\mathrm{O}_{2} \bullet\right.$ ), l'acide hypochloreux ( $\mathrm{HOCl})$, le radical peroxyle $(\mathrm{ROO} \bullet)$, le peroxynitrite (ONOO-) ainsi que l'oxyde nitrique $(\bullet N O N)[68,69,21]$.

De plus, la PC s'est montrée efficace dans l'inhibition du blanchiment du $\beta$-carotène, l'inhibition de la formation du complexe $\mathrm{Fe}^{2+}$-Ferrozine, la réduction du $\mathrm{Fe}^{3+}$ (complexe de cyanure ferrique) en forme ferreuse $\mathrm{Fe}^{2+}$, ainsi que la neutralisation du 1,1-diphényl-2picrylhydrazyle (DPPH) [14]. Ce pouvoir antioxydant de la PC est attribué, à la fois, à la chaine polypeptidique (des sous-unités $\alpha$ et $\beta$ ) et au chromophore [70]. Dans leur étude, Lissi et al., [71] ont indiqué que la neutralisation des radicaux peroxyl de la PC, dépourvue du chromophore, est comparable à celle de la PC native. De plus, Romay et al., [68] ont démontré que la chaine polypeptidique élimine les radicaux $\mathrm{HOCl}$, en réagissant avec la cystéine et les résidus de méthionine. Cependant, la PC native ne peut pas être le principal antioxydant fonctionnel in vivo. Dans son étude, McCarty [72] a montré que le principal initiateur de l'activité anti-oxydante serait la phycocyanorubine, une forme réduite de chromophore de la phycocyanine, en raison de sa similitude structurale avec 
Tableau III. Principaux effets biologiques in vivo et in vitro de la phycocyanine

\begin{tabular}{|c|c|c|c|c|c|c|}
\hline Effet biologique & Mode & Voie & PC & $\begin{array}{l}\text { Durée de } \\
\text { traitement }\end{array}$ & $\begin{array}{l}\text { Agent } \\
\text { inducteur/souches }\end{array}$ & $\begin{array}{l}\text { Réfé- } \\
\text { rences }\end{array}$ \\
\hline \multirow[t]{2}{*}{$\begin{array}{l}\text { Anti- } \\
\text { inflammatoire }\end{array}$} & $\begin{array}{l}\text { in vivo (souris OF1 } \\
\text { mâles) }\end{array}$ & peros & $\begin{array}{l}50-200 \\
\mathrm{mg} / \mathrm{kg}\end{array}$ & $1 \mathrm{~h}$ avant & $\begin{array}{l}\text { Glucose oxydase (50 } \\
\text { U) }\end{array}$ & [67] \\
\hline & $\begin{array}{l}\text { in vitro } \\
\text { (Erythrocytes) }\end{array}$ & & $10-100 \mathrm{mM}$ & $15 \mathrm{~min}$ & $\begin{array}{l}\text { AAPH }(50 \mathrm{mM}) \\
\text { durant } 4-6 \mathrm{~h}\end{array}$ & {$[20]$} \\
\hline Hépatoprotecteur & in vitro (lignée LO2) & & $\begin{array}{l}30-250 \\
\mu \mathrm{g} / \mathrm{mL}\end{array}$ & $48 \mathrm{~h}$ après & $\begin{array}{l}\mathrm{CCl} 4(10 \mathrm{mmol} / \mathrm{L}) \\
\text { durant } 6 \mathrm{~h}\end{array}$ & [73] \\
\hline Neuroprotecteur & $\begin{array}{l}\text { in vivo ( Rats Lewis } \\
\text { maâle) }\end{array}$ & IP & $25 \mathrm{mg} / \mathrm{kg}$ & 12 jours & $\begin{array}{l}\text { Encephalotogène }+ \\
\text { infection à bordetella } \\
\text { pertussis }(200 \times 10 \\
\text { UFC/ mL) }\end{array}$ & {$[76]$} \\
\hline Nephroprotecteur & $\begin{array}{l}\text { in vivo (Souris CD-1 } \\
\text { mâle) }\end{array}$ & IP & $5-30 \mathrm{mg} / \mathrm{kg}$ & $1 \mathrm{~h}$ avant & Cisplatin (18 mg/kg) & [74] \\
\hline Retinoprotecteur & $\begin{array}{l}\text { in vivo (Rat Wistar } \\
\text { mâle et femelle) }\end{array}$ & IP & $200 \mathrm{mg} / \mathrm{kg}$ & Immédiatement & $\begin{array}{l}\text { Sélénite de sodium } 19 \\
\mu \mathrm{mol} / \mathrm{kg}\end{array}$ & [77] \\
\hline Anti Atherosgène & $\begin{array}{l}\text { in vivo (Hamster } \\
\text { syérien doré) }\end{array}$ & Peros & $36 \mathrm{mg} / \mathrm{g} / \mathrm{j}$ & 12 semaines & $\begin{array}{l}\text { Régime iatrogénique } \\
\text { dépourvu en } \\
\text { Sélénium et en } \\
\text { vitamine } C \text { et } E\end{array}$ & {$[78]$} \\
\hline Antibactérien & in vitro & $\begin{array}{l}\text { Méthode } \\
\text { des puits }\end{array}$ & $5 \mathrm{mg} / \mathrm{mL}$ & $\begin{array}{l}\text { E. coli; S. aureus, } \\
\text { Pseudomonas sp }\end{array}$ & $\begin{array}{l}\text { M. luteus, } \\
\text {; K. pneumoniae }\end{array}$ & [79] \\
\hline
\end{tabular}

${ }^{1}$ Cellules granulaire cérébrales, IP : Intra-Péritonéale

tion de radicaux superoxydes, par la NADPH oxydase et peut donc jouer des rôles de protection supplémentaire, en réduisant la génération de dérivés réactifs à l'oxygène dans le corps [72].

\section{L'effet anti-inflammatoire de la PC}

Il est bien établi que la génération des ROS peut initier et engendrer des cascades inflammatoires, provoquant des lésions au niveau du foie, des reins, du cœur, etc... Plusieurs études ont montré le pouvoir de la PC dans l'atténuation du processus inflammatoire in vivo et in vitro (Tableau III). Par exemple, dans leur étude, Romay et al., [67] ont rapporté que l'effet inflammatoire de la PC avant l'induction de la réaction inflammatoire. La PC (5-30 mg/kg en intrapéritonéal) $a$, également, présenté un effet néphroprotecteur sur des souris males CD-1, ayant reçu du cisplatin (18 mg/kg) [74]. Plusieurs études ont montré que la PC inhibe les réactions inflammatoires, selon différentes voies. Les voies, les plus répandues, sont l'inhibition de l'expression de certaines enzymes impliquées dans le processus inflammatoire, telle que la cyclooxygénase-2 (COX-2), l'oxyde nitrique synthétase, la myéloperoxydase, etc...De plus, l'inhibition de la production de cytokines proinflammatoires, la réduction de la production de prostaglandine $\mathrm{E}$ et
I'inactivation du facteur nucléaire-KB (NF-KB), responsable de la dégradation de la cytokine $\operatorname{lk} B-\alpha$, ont été induites par la PC [75].

\section{Effet antitumoral de la phycocyanine}

Plusieurs études ont admis que la PC peut inhiber la prolifération des cellules cancéreuses in vitro (Tableau IV), tels que le mélanome A375 [80], l'adénocarcinome du sein MDA-MB-231 [81], les cellules cancéreuses du colon HT-29 [82], I'adénocarcinome du poumon A549 [83], les cellules cancéreuses du cerveau humain HeLa [84] et le carcinome de la prostate LNCaP [85].

Les différents travaux de recherche sur l'effet tumoral de la PC ont conclu que les principaux mécanismes impliqués seraient l'induction de la voie intrinsèque de l'apoptose ou la condensation et la fragmentation de l'ADN, empêchant, ainsi, les cellules cancéreuses de se multiplier [69]. En raison de ses propriétés antitumorales, la PC a été utilisée en tant qu'agent complémentaire aux médicaments anticancéreux traditionnels de chimiothérapie ou dans la thérapie photodynamique, en combinaison avec le laser He-Ne (hélium-néon) renforçant, ainsi, le ralentissement de la croissance des cellules cancéreuses et réduisant les effets secondaires toxiques des médicaments conventionnels utilisés en chimiothérapie [86]. 


\begin{tabular}{|c|c|c|c|c|}
\hline $\begin{array}{l}\text { Type de } \\
\text { Cancer }\end{array}$ & $\begin{array}{l}\text { Lignée } \\
\text { cellulaire }\end{array}$ & $\begin{array}{l}\text { Concentration } \\
\text { de la PC }\end{array}$ & Mécanismes /Marqueurs & Références \\
\hline \multirow[t]{2}{*}{ Peau } & A375 & $40 \mu \mathrm{M}$ & Fragmentation de I'ADN & [80] \\
\hline & & & $\begin{array}{l}\text { Perturbation du potentiel de la membrane } \\
\text { mitochondriale }\end{array}$ & \\
\hline \multirow[t]{2}{*}{ Sein } & MDA-MB-231 & $5,9 \mu \mathrm{M} / \mathrm{mL}^{\mathrm{a}}$ & Activation de la capase- 9 & [81] \\
\hline & & & libération du cytochrome c & \\
\hline Colon & HT-29 & $5 \mu \mathrm{M}^{\mathrm{b}}$ & Fragmentation de l'ADN & {$[82]$} \\
\hline \multirow[t]{3}{*}{ Poumon } & A549 & $40 \mu \mathrm{g} / \mathrm{L}^{\mathrm{c}}$ & Fragmentation de l'ADN & \\
\hline & & & Régulation du NF-kB & [83] \\
\hline & & & Augmentation du p38 MAPK & \\
\hline Prostate & LnCaP & $500 \mu g^{d}$ & Activation de la capase- 9 et -3 & {$[85]$} \\
\hline \multirow[t]{2}{*}{ Cerveau } & HeLA & $80 \mu \mathrm{g} / \mathrm{mL}$ & Activation de la capase $2,3,4,6,8,9$ et 10 & \\
\hline & & & Libération du cytochrome c & {$[84]$} \\
\hline
\end{tabular}

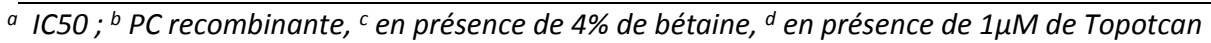

De plus, les propriétés fluorescentes de la PC, principalement, sa capacité à se lier aux cellules cancéreuses, ont permis son utilisation dans la colocalisasation des tumeurs in vivo [87].

\section{Autres effets biologique de la PC}

En plus des trois principales bioactivités de la PC décrites plus haut, d'autres activités biologiques ont été rapportées. L'étude de Gupta et al., [88] a indiqué que la $P C$ dynamise les fonctions du système immunitaire, le thymus notamment, qui joue un rôle important dans l'immunité cellulaire, favorisant l'activité des globules blancs. De plus, la PC diminue les réactions allergiques, en abaissant l'excès d'immunoglobulines $E$ (IgE) dans le sang [99]. Elle participe, également, à la protection des artères contre l'athérosclérose et réduit, significativement, le taux de lipoprotéines de faible densité (LDL) dans le sang [78]. D'autre part, la PC prévient la survenue et l'aggravation de la cataracte, provoquée par le sélénite de sodium, en bloquant l'activité de la superoxyde dismutase chloramphénicol acétyltransférase [77]. Par ailleurs, l'activité antibactérienne de la PC, étudiée selon la méthode des puits, a indiqué que la PC présente un bon effet antibactérien contre plusieurs souches bactériennes, telles qu'Escherichia coli, Staphylococcus aureus, Klepsiella pneumoniae, Pseudomonas aeroginosa, Micrococcus luteus [79, 14].

Application en nanotechnologie : Nanoparticules de PC et leurs effets biologiques

Parallèlement aux effets biologiques et pharmacoogiques (anti-tumoraux, principalement) de la PC, admi- nistrée, telle qu'elle, des travaux de recherches sont, actuellement, axés sur les nouvelles formes d'administration de la PC, afin d'améliorer et de mieux cibler son effet biologique [90]. Ainsi, l'élaboration de nanoparticules de PC a commencé à émerger. En effet, en raison de leur rapport surface sur volume élevé, les nanoparticules atteignent et agissent de manière plus efficace, au niveau de leur cible. Ces nanoparticules ont déjà prouvé leur efficacité dans les domaines thérapeutiques ou théranostiques (joignant à la fois la thérapie et le diagnostic) [91]. Ainsi, la formulation de la PC, sous forme de nanoparticules, en présence de nitrate d'argent $\left(\mathrm{AgNO}_{3}\right)$, a montré une bonne activité anti-tumorale in vitro contre le carcinome de l'ascite d'Ehrlich, comparativement aux nanoparticules d'AgNO même, l'incorporation de la PC dans une nanoformulation, à base de Paclitaxel, s'est montrée efficace contre le glioblastome humain U87MG, comparativement au paclitaxel seul [93]. De plus, l'ajout de la PC à des nanoparticules, à base de chitosane chargée de Cephalothine-bacteriocine, a permis de stabiliser et d'améliorer, à la fois, leur bio-compabilité, la libération et l'activité antibactérienne du Cephalothinebacteriocine, comparativement, aux nanoparticules sans PC [94]. L'étude de Zhao et al., [95] a indiqué que la présence de $P C$ dans des nanomicelles de dextrane, chargée de cordycepine, a permis d'augmenter leur effet antiprolifératif contre les cellules cancéreuses du cerveau $\mathrm{C6}$, comparativement à la $\mathrm{PC}$ et à la cordycepine, testées séparément. Bharathiraja et al., [96] ont élaboré des nanoparticules, à base d'albumine de sérum bovin et de PC. Les nanoparticules obtenues, en combinaison avec une lumière laser, ont, 
efficacement, éradiqué les cellules de l'adénocarcinome du sein humain, où la $\mathrm{PC}$ a permis de générer des dérivés réactifs à l'oxygène et de convertir l'énergie op-tique absorbée en une énergie thermique, responsable de l'éradication de ces cellules cancéreuses. De plus, la présence de la PC a permis aux nanoparticules de générer une bonne amplitude de signaux ultrasonores sous un système d'imagerie photoacoustique qui a amélioré la résolution de l'imagerie des cellules traitées. En outre, les nanoparticules de sélénium, fonctionnalisées par la $P C$, ont bloqué le processus apoptotique des cellules pancréatiques $\beta$, induisant le diabète [97]. L'ajout de PC aux chitosomes, à base de mélange entre le chitosane et le xanthane, produits par atomisation, a permis l'obtention de chitosomes, hautement muco-adhésifs, servant à prévenir les maladies relatives au dysfonctionnement du colon [98].

\section{Conclusions et perspectives}

La recherche de nouvelles sources potentielles et efficaces de biomolécules, pour différentes applications biologiques et pharmacologiques, a fait l'objet d'efforts considérables, au cours de ses dernières années. Ainsi, les microalgues, de par les avantages de croissance rapide qu'elles présentent, ont été le sujet de plusieurs études de recherche, en particulier, pour certains de leurs métabolites uniques inexistants chez les végétaux supérieurs. II s'agit des molécules présentes dans le phycobilisome des microalgues, dont la phycocyanine, qui a suscité le plus d'intérêt, en raison de son caractère hydrophile, non-toxique, ainsi que son large spectre d'activités biologiques et pharmacologiques. Bien que la phycocyanine ait, déjà, montré un grand potentiel d'utilisation, une partie de ce potentiel bio-actif n'est, certainement, pas encore totalement ex-ploité, en raison des limitations rencontrées lors de sa production et sa purification combinées aux limita-tions liées à son caractère thermosensible. Ainsi, il est vraisemblable que dans les années à venir, des étu-des sur l'optimisation des processus biotechnolo-giques de production et de purification de la PC se-ront amplifiées, dans le but de multiplier son utilisa-tion à grande échelle. De plus, il serait possible d'a-xer, en amont, ces études sur le screening ciblé de microalgues et $d$ 'isoler des souches contenant des te-neurs élevées en PC pouvant, même, présenter des caractéristiques de thermorésistance intéressantes, autres que celles déjà disponibles sur le marché ac-tuel, ce qui va contribuer à l'évolution de l'application de la PC, sans contraintes en industrie. Par ailleurs, vu l'absence de données, portant sur le mécanisme de thermostabilisation de la PC, à travers l'ajout d'agents stabilisants conventionnels, il est important d'étudier, en profondeur, les mécanismes impliqués dans la thermostabilisation de la PC et prendre en compte les différents aspects, notamment, structuraux, optiques et thermodynamiques. Parallèlement à la stabilisation chimique, l'optimisation de l'encapsulation de la PC, tout en considérant plusieurs facteurs, en conditions industrielles, est un axe de recherche très important qui permettra de garantir une meilleure stabilité et une longue durée de vie de la PC, dans l'état le plus natif possible, de modéliser son activité mais, aussi, d'offrir une nouvelle forme de commercialisation de la $P C$ qui serait plus compétitive.

Par ailleurs, depuis 1998, le potentiel biologique de la $P C$ a été mis en évidence. De nombreuses études appuient le fait que la PC pourrait être un principe actif très efficace, surtout, pour réduire les tumeurs. Compte tenu de ces résultats, les études sur les activités biologiques de la PC se sont multipliées et sont arrivées à exploiter la $P C$ dans la thérapie ciblée, à travers l'élaboration de nanoparticules. Cependant, d'autres travaux sont, encore, nécessaires afin d'optimiser les conditions d'élaboration de ces nanoparticules de PC, en essayant plusieurs matériaux, tout en étudiant leur efficacité de délivrance. $D^{\prime}$ autre part, la combinaison de la PC, avec d'autres molécules antitumorales ou radiations, est devenue un autre axe de recherche, étant donné que les médicaments, traditionnellement utilisés en chimiothérapie, ont des effets secondaires très néfastes pour les malades. Ainsi, I'incorporation de la PC dans ces médicaments ou avec les radiations pourrait réduire ces effets et améliorer l'effet thérapeutique contre les tumeurs. Bien que certains mécanismes de la bioactivité de la PC aient été élucidés, d'autres études sont, encore, nécessaires, afin de mieux investiguer les mécanismes moléculaires de la PC, surtout, les effets antiinflammatoires et anti-tumoraux. Des travaux de recherche sur l'élucidation des mécanismes moléculaires thérapeutiques de la PC sont, également, nécessaires afin de permettre l'utilisation de la PC en tant que médicament.

\section{Conflit d'intérêts}

Les auteurs ne déclarent aucun conflit d'intérêt.

\section{Références}

1. Rabiei Z., Solati K., Amini-Khoei H. Phytotherapy in treatment of Parkinson's disease : a review. 
Pharma Bio 2019; 51:355-62.

2. Ferreira TS., Moreira CZ., Cária NZ., Victoriano G., Silvra W., Magalhães J. Phytotherapy : an introduction to its history, use and application. Rev Bras Plant Med 2014; 16:290-8.

3. Chentir I., Doumandji A., Ammar J., Zili F., Jridi M., Markou G., Ouada HB. Induced change in Arthrospira sp. (Spirulina) intracellular and extracellular metabolites using multifactor stress combination approach. J Appl Phycol 2018; 30: 1563-74.

4. Markou G., Nerantzis E. Microalgae for high-value compounds and biofuels production: a review with focus on cultivation under stress conditions. Biotechnol Adv 2013; 31:1532-42.

5. Manirafasha E., Ndikubwimana T., Zeng X., Lu Y., Jing K. Phycobiliprotein: potential microalgae derived pharmaceutical and biological reagent. Biochem Eng J 2016; 109:282-96.

6. Pagels F., Guedes A., Amaro H., Kijjoa A., Vasconcelo V. Phycobiliproteins from cyanobacteria: Chemistry and biotechnological applications. Biotech Adv 2019; 37:422-43.

7. Sinha R., Lebert M., Kumar A., Kumar H., Häder $D-P$. Spectroscopic and biochemical analyses of UV effects on phycobiliproteins of Anabaena sp. and Nostoccarmium. Bot Acta 1995; 180:87-92.

8. Vonshak A. Spirulina platensis (Arthrospira): physiology, cellbiology and biotechnology, In Vonshak A, Ed. Taylor \& Francis, London; 2002, p 4365.

9. Sekar S., Chandramohan M. Phycobiliproteins as a commodity: trends in applied research, patents and commercialization. J Appl Physiol 2008; 20: 113-6.

10. de Morais M., Fontoura Prates D., Botelho Moreira J., Hartwig Duarte J., Vieira Costa J. Phycocyanin from Microalgae: properties, extraction and purification, with some recent applications. Indust Biotech 2018;14: 30-7.

11. Jiang L., Wang Y., Yin Q., Liu G., Liu Y., Huang H., Li B. Phycocyanin: A potential drug for cancer treatment. J Can 2017; 8:3416-29.

12. Zheng Y., Mo L., Zhang W., Duan Y., Huang Y., Chen C. et al. Phycocyanin fluorescent probe from Arthrospira Platensis: preparation and application in LED-CCD fluorescence density strip qualitative detection system. J Appl Phycol 2019; 31:1107-15.

13. Najafian Y., Hamedi S., Farshchi M., Feyzabadi Z. Plantago major in Traditional Persian Medicine and modern phytotherapy: a narrative review. Elect Phys 2018; 10:6390-9.
14. Chentir I., Hamdi M., Suming L., Doumandji A., Markou G., Nasri M. Stability, bio-functionality and bio-activity of crude phycocyanin from a two-phase cultured Saharian Arthrospira sp. strain. Alg Res 2018; 35:395-406.

15. Kannaujiya V., Sinha R. Thermokinetic stability of phycocyanine and phycoerythrin in food-grade presrvatives. J Appl Phycol 2016; 28:1063-70.

16. Yan M., Liu B., Jiao X., Qin S. Preparation of phycocyanin microcapsules and its properties. Food Bioprod Proces 2014; 92:90-7.

17. Arteni A., Ajlani G., Boekema E. Structural organisation of phycobilisomes from Synechocysti ssp. strain PCC6803 and their interaction with the membrane. Biochim Biophys Acta BBA -Bioenerg 2009; 10787: 272-9.

18. Boussiba S., Richmond A. Isolation and characterization of phycocyanin from the blue-green alga Spirulina platensis. Arch Microb 1979; 120: 155-9.

19. Glazer A. Phycobiliproteins-A family of valueble, widely used fluorophore. J Appl Phycol 1994; 6:105-12.

20. Benedetti S., Benvenuti F., Pagliarani S., Francogli S., Scoglio S., Canestrari F. Antioxidant properties of a novel phycocyanin extract from the blue-green alga Aphanizomenon flosaquae. Life Sci 2004; 75:2353-62.

21. Fernández-Rojas B., Hernández-Juárez J., Pedraza-Chaverri J. Nutraceutical properties of phycocyanin. J Funct Foods 2014; 11: 375-92.

22. BCC. Buisness communication company Phycocyanin Market: Food \& Beverage Application to Hold Close to $85 \%$ Value Share Throughout the Forecast Period: Global Industry Analysis (20132017) \& Opportunity Assessment (2018 - 2028)https://www.futuremarketinsights.com/reports/ phycocyanin-market-

23. Kilimtzidi E., Cuellar Bermudez S., Markou G., Goiris K., Vandamme D., Muylaert K. Enhanced phycocyanin and protein content of Arthrospira by applying neutral density and red light shading filters: a small-scale pilot experiment. J Chem Technol Biotechnol 2019; 94:2047-54.

24. Hifney A., Issa A., Fawzy M. Abiotic stress induced production of $\beta$-carotene, allophycocyanin and total lipids in Spirulina sp. J Biol Earth Sci 2013;3:54-64.

25. Setyoningrum T., Nur A. Optimization of c-phycocyanin production from $S$. platensis cultivated on mixotrophic condition by using response surface methodology. Biocat Agric Biotech 2015; 4: 603-7. 
26. Bachchhav M., Kulkarni M., Ingale A. Enhanced phycocyanin production from Spirulina platensis using light emitting diode. J Instit Eng (India): Series E 2017;92: 41-5.

27. Patel V., Berthold D., Dhandayuthapani S., Rathinavelu A., Gantar M. Optimization of C-phycocyanin production by Limnothrix sp. 37-2-1. Alg Biomass UtIn 2016; 7:104-11.

28. Mogany T., Swalaha F., Kumari S., Bux F. Elucidating the role of nutrients in C-phycocyanin production by the halophilic cyanobacterium Euhalothece Sp. J App Phycol 2018; 30:2259-571.

29. Deshmukh D., Puranik P. Statistical evaluation of nutritional components impacting phycocyanin production in Synechocystis sp. Braz J Microbio 2012; 43:348-55.

30. Kuddus M., Singh P., Thomas G., Al-Hazimi A. Recent developments in production and biotechnological applications of C-phycocyanin. Bio Med Res Int 2013; 2013:1-9.

31. Moraes C., da Costa Ores J., Costa J., Kalil S. Recovery of c-phycocyanin in the presence of cells using expanded bed IEC. Chromat 2011; 74:30712.

32. Kannaujiya V., Sinha R. An efficient method for separation and purification of phycobiliproteins from rice-field cyanobacterium Nostoc sp. strain HKAR-11. Chromato 2016; 76:335-43.

33. Sarada R., Pillai M., Ravishankar G. Phycocyanin from Spirulina sp: influence of processing of biomass on phycocyanin yield, analysis of efficacy of extraction methods and stability studies on phycocyanin. Process Biochem 1999; 34:795-801.

34. Bhaskar U., Gopalswamy G., Raghu R. Simple method for efficient extraction and purification of C-Phycocyanin from Spirulina platensis Geitler. Ind J Exp Biol 2005; 43:277-9.

35. Viskari P., Colyer C. Rapid extraction of phycobiliproteins from cultured cyanobacteria samples. Anal Biochem 2003; 319 :263-71

36. Martínez J., Luengo E., Saldaña G., Álvarez I., Raso J. C-phycocyanin extraction assisted by pulsed electric field from Artrosphira platensis. Food Res Int 2017; 99:1042-7.

37. Aftari R., Rezaei K., Bandani A., Mortazavi A. Antioxidant activity optimization of Spirulina platensis C-phycocyanin obtained by freeze-thaw, microwave-assisted and ultrasound-assisted extraction methods. Qual Assur Saf Crops Foods 2017; 9:1-99.

38. Dejsungkranont M., Chen H-H., Sirisansaneeyakul S. Enhancement of antioxidant activity of Cphycocyanin of spirulina powder treated with supercritical fluid carbon dioxide. Agri Nat Res 2017; 51:347-54.

39. Kumar D., Dhar D., Pabbi S., Kumar N., Walia S., Extraction and purification of C-phycocyanin from Spirulina platensis (CCC540). Indian J Plant Physiol 2014; 19:184-8.

40. Patil G., Chethana S., Sridevi A., Raghavarao K. Method to obtain C-phycocyanin of high purity. J Chromatogr A 2006; 1127:76-81.

41. Rito-Palomares M., Nunez L., Amador D. Practical application of aqueous two-phase systems for the development of a prototype process for C-phycocyanin recovery from Spirulina maxima. J Chem Technol Biotechnol 2001; 76:1273-80.

42. Benedetti S.,Rinalducci S., Benvenuti F., Francogli S., Pagliarani S., Giorgi L. et al. Purification and characterization of phycocyanin from the blue-green alga Aphanizomenon flos-aquae. I Chromato B Analyt Technol Biomed Life Sci 2006; 20:12-8.

43. Wang X., Li L, Chang W., Zhang J., Gui L., Gua B., Liang D. Structure of C-phycocyanin from Spirulina platensis at 2.2A resolution: a novel monoclinic crystal form for phycobiliproteins in phycobilisomes. Acta Crystallog D 2001; 57: 784-92.

44. Antelo F., Costa J., Kalil S. Thermal degradation kinetics of the phycocyanin from Spirulina platensis. Biochem Eng J 2008; 41:43-7.

45. Chaiklahan R., Chirasuwan N., Bunnag B. Stability of phycocyanin extracted from Spirulina sp.: influence of temperature, $\mathrm{pH}$ and preservatives. Process Biochem 2012; 47:659-64.

46. Rastogi R., Sonani R., Madamwar D. Physico-chemical factors affecting the in vitro stability of phy -cobiliproteins from Phormidium rubidum A09 DM. Bioresour Technol 2015; 190:219-26

47. Martelli G., Folli C., Visai L., Daglia M., Ferrari D. Thermal stability improvement of blue colorant C-phycocyanin from Spirulina platensis for food industry applications. Process Biochem 2014; 49: 154-9.

48. Fukui K., Saito T., Noguchi Y., Kodera Y., Matsushima A., Nishimura H., Inada Y. Relationship between color development and protein conformation in the phycocyanin molecule. Dyes Pigm 2004; 63:89-94.

49. Kikuchi H., Sugimoto T., Mimuro M. An electronic state of the chromophore, phycocyanobilin, and its interaction with the protein moiety in C-phycocyanin: protonation of the chromopho -re. Chem Phys Lett 1997; 247:460-5.

50. Christwardana M., Sutanto H., Suzery M., Amelia D., Aritonang R. Kinetic study on the effects 
of sugar addition on the thermal degradation of phycocyanin from Spirulina sp. Food Biosci 2018; 22:85-90.

51. Pan-utai W., lamtham S. Physical extraction and extrusion entrapment of C-phycocyanin from $\mathrm{Ar}$ throspira platensis.J King Saud University-Science 2018.https://doi.org/10.1016/j.jksus.2018.05.06.

52. Hadiyanto M., Majid D., Setyawan D., Sutanto H., Improvement of stability and antioxidant activeties by using phycocyanin-chitosan encapsulation technique.IOP Conf Ser: Earth Environ Sci 2017; 55:1-9.

53. Braga A., da Silva Figueira F., da Silveira J., de Morais G., Vieira Costa J.,Kalil S. Improvement of thermal stability of C-phycocyanin by nanofi-ber and preservative agents: c-phycocyanin stability : preservative and nanofiber. J Food Proc 2016; 40:1264-9.

54. Kepekçi R., İçoğlu H., Kireçci A. Assessment of antioxidant activity and phycocyanin release of spirulina loaded poly ( $\varepsilon$-caprolactone) electrospun nanofibres. T He J Text Instit 2017; 108: 1840-6.

55. Yagoubi S., Shahidi F., Mohebbi M., Varidi M., Golmohammadzadeh S. Preparation, characterization and evaluation of physicochemical properties of phycocyanin-loaded solid lipid nanoparticles and nanostructured lipid carriers, J Food Measur Charact 2018; 12:378-85.

56. HadiyantoM., Suzery M., Setyawan D., Majid D., Sutanto H. Encapsulation of phycocyanin-alginate for high stability and antioxidant activety. $1 O P$ Conf Ser: Earth and Environ Sci 2017; 55012030.

57. Dewi EN., Purnamayati L., Kurniasih RA. Physical characteristics of phycocyanin from spirulina microcapsules using different coating materials with freeze drying method.IOP Con Ser: Earth and Enviro Sci 2017; 55012060.

58. Selig M., Malchione N., Amaleldin S., Padilla-Zakour O., Abbaspourrad A. Protection of blue color in a Spirulina derived phycocyanin extract from proteolytic and thermal degradation via complexation with beet-pectin. Food Hydrocol 2018; 74:46-52.

59. Chen X., Wu M., Yang Q., Wang S. Preparation, characterization of food grade phycobiliproteins from Porphyra haitanensis and the application in liposome-meat system.LWT Food Sci Technol 2017; 77:468-74.

60. Batista A., Raymundo A., Sousa I., Empis J. Rheological characterization of coloured oil-in-water food emulsions with lutein and phycocyanin added to the oil and aqueous phases. Food Hydro- col 2006; 20: 44-52.

61. Mohammadi-Gouraji E., Soleimanian-Zad S., Ghiaci $M$. Phycocyanin-enriched yogurt and its antibacterial and physicochemical properties during 21 Days of storage. LWT Food Sci Technol 2019; 102: 230-6.

62. Chentir I., Kchaou H., Hamdi H., Jridi M., Li S., Doumandji A., Nasri M. Biofunctional GelatinBased Films Incorporated with Food Grade Phycocyanin Extracted from the Saharian Cyanobacterium Arthrospira Sp. Food Hydrocol 2019; 89: 715-25.

63. Simis S., Peters S., Gons H. Remote sensing of the cyanobacterial pigment phycocyanin in turbid inland water. Limno Oceano 2005;50:237-45.

64. Badakere SS., Chablani AT., Bhatia HM., Perjwani DP., Mehta BC. Recent Trends in Immunology. Institute of Immunohaematology Publication, ICMR, Mumbai, India, 1982.

65. Gregor J., Marsalek B., Sipkova H. Detection and estimation of potentially toxic cyanobacteria in raw water at the drinking water treatment plant by in vivo fluorescence method. Water Res 2007; 41:228-34.

66. Durackova Z. Some current insights into oxidative stress. Physio Res 2010; 59:459-69.

67. Romay C., Armesto J., Remirez D., Gonzalez R., Ledon N., García I., Antioxidant and anti-inflammatory properties of C-phycocyanin from bluegreen algae. Inflam Res 1998; 47:36-41

68. Romay C., Gonzalez R., Pizarro M., Lissi E. Kinetics of C-phycocyanin reaction with hypochlorite. J Prot Chem 2000; 19:151-5.

69. Thangam R., Suresh V., Asenath Princy W., RajkumarM., Senthilkumar N., Gunasekaran P.et al. C-Phycocyanin from Oscillatoria tenuis exhibited an antioxidant and in vitro antiproliferative activity through induction of apoptosis and G0/G1 cell cycle arrest. Food Chem. 2013; 140: 262-72.

70. Pleonsil P., Soogarun S., Suwanwong Y. Antioxidant activity of holo- and apo-c phycocyanin and their protective effects on human erythrocytes. Int J Biol Macromol 2013; 60:393-8.

71. Lissi E., Pizarro M., Aspee A., Romay C. Kinetics of phycocyaninebilin groups destruction by peroxyl radicals. Free Rad Biol Med 2000; 28: 1051-5.

72. McCarty MF. latrogenic Gilbert syndrome-a strategy for reducing vascular and cancer risk by increasing plasma unconjugated bilirubin. Med Hypotheses 2007; 69:974-94.

73. Ou Y., Zheng S., Lin L., Jiang Q., Yang X. Protective effect of $C$-phycocyanin against carbon te- 
trachloride-induced hepatocyte damage in vitro and in vivo. Chem Biol Interact 2010; 185:94-100.

74. Fernandez-Rojas B., Medina-Campos O., Hernandez-Pando R., Negrette-Guzman M., HuertaYepez S., Pedraza-Chaverri J. C-phycocyanin prevents cisplatin-induced nephrotoxicity through inhibition of oxidative stress. Food Funct 2014; 5:480-90.

75. Soni A., Dubey M., Verma M., Dhankhar R., Kaushal V., Atri R., Sabharwal R. Revisiting the role of phycocyanin in current clinical practice. IJ $P S$ $R$ 2015; 6:4588-600.

76. Pentón-Rol G., Martínez-Sanchez G., CervantesLlanos M., Lagumersindez-Denis N., Acosta-Medina EF., Falcón-Cama V. et al. C-phycocyanin ameliorates experimental autoimmune encephalomyelitis and induces regulatory $\mathrm{T}$ cells. Inter Immunopharma 2011; 11:29-38.

77. Kumari R., Anbarasu K. Protective role of C-phycocyanin against secondary changes during sodium selenite mediated cataractogenesis. Nat Prod Biopros 2014; 4:81-9.

78. Riss J., Décordé K., Sutra T., Delage M., Baccou JC., Jouy N. et al. Phycobiliprotein C-phycocyanin from Spirulina platensis is powerfully responsible for reducing oxidative stress and NADPH oxidase expression induced by an atherogenic diet in hamsters. J Agric Food Chem 2007; 55:7962-7.

79. Sarada D., Kumar SC., Rengasamy R. Purified Cphycocyanin from Spirulinaplatensis (Nordstedt) Geitler: a novel and potent agent against drug resistant bacteria. W J Microbiol Biotechnol 2011; 7:779-83.

80. Chen T., Wong YS. In vitro antioxidant and antiproliferative activities of selenium-containing phycocyanin from selenium-enriched Spirulina platensis. J Agri Food Chem 2008; 56:4352-8.

81. Ravi M., Tentu S., Baskar G., Rohan Prasad S., Raghavan S., Jayaprakash P. et al. Molecular mechanism of anti-cancer activity of phycocyanin in triple-negative breast cancer cells. BMC Cancer 2015; 15:768-81.

82. Wang H., Li Y., Gao X., Carter C., Liu Z. The recombinant beta subunit of C-phycocyanin inhibits cell proliferation and induces apoptosis. Cancer Let 2007; 247:150-8.

83. Bingula R., Dupuis C. Study of the effects of betaine and/or C-phycocyanin on the growth of lung cancer A549 cells in vitro and in vivo. J Oncol 2016; 2016:1-11.

84. Li B., Gao MH., Zhang XC., Chu XM. Molecular immune mechanism of C-phycocyanin from Spirulina platensis induces apoptosis in HeLa cells in vitro. Biotechnol Appl Biochem 2006; 43:155-64.

85. Gantar M., Simovic D., Djilas S., Gonzalez WW., Miksovska J. Isolation, characterization and antioxidative activity of C-phycocyanin from Limnothrix sp. strain 37-2-1. J Biotech 2012; 159:21-6.

86. Wang CY., Wang X., Wang Y., Zhou T., Bai Y., Li YC., Huang B. Photosensitization of phycocyanin extracted from Microcystis in human hepatocellular carcinoma cells: implication of mitochondria-dependent apoptosis. J Photochem Photobiol B Biology 2012; 117:70-9.

87. Morcos N., Berns M., Henry W., Phycocyanin: Laser activation, cytotoxic effects, and uptake in human atherosclerotic plaque. Laser Surg Med 1988; 8:10-7.

88. Gupta M., Dwivedi UN., Khandelwal S. C-phycocyanin: An effective protective agent against thymic atrophy by tributyltin. Toxic Let 2011; 204:211.

89. Remirez D., Ledón N., González R. Role of Histamine in the inhibitory effect of phycocyanin in experimental models of allergic inflammatory response. Mediat Inflamm 2002; 11:81-5.

90. Zhao X., Zhao X., Yuan M., Han R., Lian J., Zhao $X$. et al. Study on physisorption between phycocyanin and gold nanoparticles. Luminescence 2019;34:623-7.

91. Chuto G., Chaumet-Riffaud P., Les nanoparticules. Médecine Nucléaire 2010;34:370-6.

92. El-Naggar NEA. Hussein MH., Atallah El-Sawah A. Bio-fabrication of silver nanoparticles by phycocyanin, characterization, in vitro anticancer activity against breast cancer cell line and in vivo cytotxicity. Sci Rep 2017;7:10844.

93. Agrawal M., Yadav SK., Agrawal SK., Karmakar S. Nutraceutical phycocyanin nanoformulation for efficient drug delivery of paclitaxel in human glioblastoma U87MG cell line. J Nano Res 2017; 19:272-81.

94. Namasivayam KR. Phycocyanin stabilized chitosan nanoparticles loaded cephalothin-bacteriocinnano drug conjugate preparation for the enhanced antibacterial activity, controlled drug release and biocompatibility. Asian J Pharm 2016 ;10(4).891.

95. Zhao M., Chen L., Chen W., Meng Z., Hu K., Du S. et al. Packaging cordycepinphycocyanin micelles for the inhibition of brain cancer. J Mat Chem $B$ 2017; 5:6016-26.

96. Bharathiraja S., Manivasagan P., Moorthy M., BuiN., Jang B., Phan T. et al. Photo-based PDT/ PTT dual model killing and imaging of cancer cells using phycocyanin-polypyrrole nanoparti- 
cles. Euro J Pharma Biopharma 2018; 123:20-30.

97. Liu Q., Huang Y., Zhang R., Cai T., Cai Y. Medical application of Spirulina platensis derived cphycocyanin. Evidence-Based Complem Alternat Med 2016:2016:1-14.
98. Manconi M., Mura S., Manca ML., Fadda A., Dolz M., Hernandez M. et al. Chitosomes as drug delivery systems for c-phycocyanin: preparation and characterization. Inter J Pharma 2010; 392(12):92-100. 